\title{
UN PROBLEMA CRÍTICO: LA MALNUTRICIÓN INFANTIL EN BOLÍVAR
}

\author{
A CRITICAL PROBLEM: THE CHILD MALNUTRITION IN BOLÍVAR
}

\section{Jairo Israel Rivera Vásquez ${ }^{(1)}$; Stephany Carolina Olarte Benavides ${ }^{(2)}$; Nadya Ximena Rivera Vásquez ${ }^{(3)}$}

(1) Universidad Andina Simón Bolivar, Av. Toledo, Quito, Ecuador

(2) Universidad Técnica Particular de Loja, Av. 6 de diciembre, Quito, Ecuador

(3) Universidad UTE, Av. Occidental, Quito, Ecuador

Email.: jairo.rivera@uasb.edu.ec

\section{DOI: https://doi.org/10.33789/talentos.8.1.147}

\begin{abstract}
Resumen: Bolivar es una provincia que se ubica en la sierra centro del Ecuador y presenta un panorama crítico en cuanto a la malnutrición infantil. El objetivo de esta investigación es analizar los determinantes de la malnutrición infantil en esta provincia. La metodología utilizada es cuantitativa, aplicada en las Encuestas de Condiciones de vida 1999-2006-2014, a través de un análisis de regresión probabilística en este estudio de tipo descriptivo. Los principales resultados del estudio encuentran que la desnutrición y el sobrepeso se asocian a distintos factores, sociales y personales, los cuales varían en el tiempo. Por una parte, entre los determinantes de la desnutrición infantil está la pobreza, el bajo peso y la educación de la madre. Por su lado, entre los determinantes del sobrepeso infantil está la edad, el carné de salud, si la madre trabaja y el abastecimiento de agua. Con ello, la principal conclusión del estudio es que la malnutrición en Bolivar tiene determinantes heterogéneos que deben ser analizados para el desarrollo de políticas públicas.
\end{abstract}

Palabras Clave: desnutrición infantil, sobrepeso infantil, Bolívar, Ecuador.

Recibido: 18 de noviembre de 2020

Aceptado: 01 de junio de 2021

Publicado como artículo científico en la Revista de Investigación Talentos VIII (1), 101-111 
Abstract: Bolivar is a province located in the central highlands of Ecuador and presents a critical panorama regarding child malnutrition. The objective of this research is to analyze the determinants of child malnutrition in this province. The methodology used is quantitative, applied in the Surveys of Living Conditions 1999-2006-2014, through a probabilistic regression analysis in this descriptive study. The main results of the study find that malnutrition and overweight are associated with different factors, social and personal, which vary over time. On the one hand, among the determinants of child undernutrition are poverty, low weight and the mother's education. On the other hand, among the determinants of child overweight are age, the health card, mother works and the water supply. With this, the main conclusion of the study is that malnutrition in Bolvar has heterogeneous determinants that must be analyzed for the development of public policies.

Keywords: child malnutrition, child overweight, Bolivar, Ecuador.

\section{INTRODUCCIÓN}

La Organización de las Naciones Unidas plantea como metas mundiales los Objetivos de Desarrollo Sostenible (ODS) 2030. El objetivo 2 de los ODS es poner fin al hambre, lograr la seguridad alimentaria, la mejora de la nutrición y promover la agricultura sostenible (ONU 2015); en ese sentido, a nivel mundial los países se encuentran realizando esfuerzos para mejorar sus indicadores nutricionales. Alrededor del $22 \%$ de los menores de cinco años sufren desnutrición crónica infantil (como retraso en el crecimiento) y cerca de $6 \%$ tienen sobrepeso infantil (como exceso de peso para la edad y estatura) (ONU 2019).

La malnutrición infantil es un problema que combina afectaciones tanto por desnutrición y sobrepeso. Esto ocasiona dificultades en el desarrollo de los niños en el corto, mediano y largo plazo, siendo una de las principales causas de muerte infantil (Cauldfield et al. 2004; Martorell 1999). Entre las principales causas de la desnutrición se encuentran factores inmediatos como enfermedades y dieta, factores subyacentes como la inseguridad alimentaria, y factores básicos como problemas estructurales en lo social y económico (Gillespie et al. 2003; UNICEF 2013). Entre las principales causas del sobrepeso se encuentran el acceso a alimentos nutritivos, el entorno de obesidad, los hábitos de consumo, y la práctica de deporte (Rivera 2012; WHO 2000).

En América Latina y el Caribe, existe una doble carga nutricional que se relaciona con esa coexistencia de desnutrición y sobrepeso a nivel individual, del hogar y del país (Rivera et al. 2014). En medio de la diversidad de prevalencias de desnutrición y sobrepeso en los países latinoamericanos, se resalta Ecuador como un caso preocupante, ya que para el año 2018, la desnutrición afecta a un $23 \%$ de la población menor de cinco años y el sobrepeso afecta a cerca de $16 \%$. En el país, durante las dos últimas décadas ha existido una limitada reducción de la desnutrición y al mismo tiempo ha habido un incremento del 
sobrepeso infantil (Gutiérrez et al. 2017).

Dentro del Ecuador, la provincia de Bolívar representa un caso crítico (MCDS 2010). La desnutrición infantil, a pesar de tener una reducción constante en el tiempo, todavía se mantiene entre las más altas del país, siendo $35 \%$ para el año 2018. El sobrepeso infantil ha incrementado constantemente en el período de análisis y se ubica sobre el promedio nacional, alcanzando 18\% para el año 2018. El objetivo de esta investigación es analizar la malnutrición infantil en la provincia de Bolívar y sus determinantes.

\section{MATERIALES Y MÉTODOS}

Para el presente estudio se utilizan las Encuestas de Condiciones de Vida (ECV) de los años 1999, 2006 y 2014. Estas encuestas fueron producidas por el Instituto Nacional de Estadísticas y Censos, y tienen representatividad nacional, área, región y provincia. A su vez, se incorpora como contexto las cifras de la provincia obtenidas de la Encuesta Nacional de Salud y Nutrición 2012 y 2018.

El tamaño de la muestra para menores de cinco años fue de 3173 niños en 1999, de 6244 niños en 2006 y de 11473 niños en 2014 (INEC 2015). Las ECV como fuente de información tienen potencialidades en referencia al tipo de datos recolectados. En ese sentido, maneja información referente a la realidad social y económica de los menores de cinco años y sus familias. Además, las bases son de libre acceso dentro del INEC.

El estudio es de tipo descriptivo. En la estimación se usa un modelo probabilístico para cada periodo, uno para desnutrición y uno para sobrepeso. El objetivo de los modelos es estimar los determinantes de la malnutrición y sus probabilidades de ocurrencia. Para ello, se usa el software STATA, y las curvas de crecimiento de la OMS (WHO 2006). Con ello, se estima el modelo:

donde $\mathrm{Y}$ es una variable binaria que toma el valor de 1 si la persona tiene desnutrición crónica en el modelo para desnutrición y 0 si no tiene desnutrición. A su vez, la variable es 1 si la persona tiene sobrepeso en el modelo para sobrepeso y 0 si la persona no tiene. Dentro de las variables explicativas se encuentran: es un vector de variables ambientales que incluye: área; es un vector de variables sociales que incluye: pobreza por consumo, agua por red pública, servicio sanitario y alcantarillado, recolección de basura, tipo de techo, tipo de paredes, tipo de piso, recibe vitamina A, y recibe hierro; es un vector de variables personales que incluye: sexo, edad, bajo peso, presencia de diarrea, presencia de enfermedades respiratorias, carné de salud, matrícula en guardería, prácticas de lactancia correctas, cuidado de la madre, trabajo madre, deporte madre, orden de nacimiento, edad de la madre y educación de la madre; con sus respectivos parámetros, .

Las estadísticas descriptivas de la provincia de Bolívar, en base a las ECV, se presenta en la Tabla 1. Bolívar es una provincia mayoritariamente rural, con cerca de tres tercios de la población en esa área, lo cual coincide con estudios previos (Gobierno de la provincia de Bolívar 2015). En el tiempo, los indicadores de bienestar dentro de la provincia han mejorado, aunque todavía 
existen necesidades insatisfechas (INEC recolección de basura; además, ha existido 2014). La pobreza por consumo se ha reducido paulatinamente, aunque todavía es superior al promedio nacional (MCDS 2017). Las coberturas de servicios se han incrementado en agua, alcantarillado y una disminución de enfermedades como diarrea y enfermedades respiratorias; $y$ se ha incrementado la obtención del carné de salud, matrícula en la guardería y de los años de educación de la madre.

Tabla 1.- Estadísticas descriptivas

\begin{tabular}{|c|c|c|c|c|}
\hline \multirow[b]{2}{*}{ Variables } & & \multicolumn{3}{|c|}{ Años } \\
\hline & & 1999 & 2006 & 2014 \\
\hline \multirow[t]{2}{*}{ Área rural } & Sí & 85.7 & 90.1 & 75.1 \\
\hline & No & 14.3 & 9.9 & 24.9 \\
\hline \multirow[t]{2}{*}{ Pobreza consumo } & Sí & 78.0 & 77.5 & 53.9 \\
\hline & No & 22.0 & 22.5 & 46.1 \\
\hline \multirow[t]{2}{*}{ Agua red pública } & Sí & 14.2 & 21.1 & 53.7 \\
\hline & No & 85.8 & 78.9 & 46.3 \\
\hline \multirow[t]{2}{*}{ Servicio sanitario y alc. } & Sí & 16.0 & 19.7 & 35.7 \\
\hline & No & 84.0 & 80.3 & 64.3 \\
\hline \multirow[t]{2}{*}{ Recolección basura mun. } & Sí & 14.2 & 24.7 & 52.2 \\
\hline & No & 85.8 & 75.4 & 47.8 \\
\hline \multirow[t]{2}{*}{ Techo adecuado } & Sí & 20.4 & 20.0 & 37.1 \\
\hline & No & 79.6 & 80.0 & 62.9 \\
\hline \multirow[t]{2}{*}{ Paredes adecuadas } & Sí & 29.5 & 46.3 & 71.3 \\
\hline & No & 70.5 & 53.7 & 28.7 \\
\hline \multirow[t]{2}{*}{ Piso adecuado } & Sí & 9.4 & 6.5 & 18.9 \\
\hline & No & 90.6 & 93.5 & 81.1 \\
\hline \multirow[t]{2}{*}{ Vitamina A } & Sí & 1.4 & 37.8 & 64.4 \\
\hline & No & 98.6 & 62.2 & 35.6 \\
\hline \multirow[t]{2}{*}{ Hierro } & Sí & 8.1 & 21.7 & 59.8 \\
\hline & No & 91.9 & 78.3 & 40.2 \\
\hline \multirow[t]{2}{*}{ Mujer } & Sí & 51.4 & 46.3 & 45.8 \\
\hline & No & 48.6 & 53.7 & 54.3 \\
\hline \multirow[t]{2}{*}{ Bajo peso } & Sí & 0.9 & 1.3 & 7.9 \\
\hline & No & 99.1 & 98.7 & 92.1 \\
\hline \multirow[t]{2}{*}{ Diarrea } & Sí & 23.7 & 29.7 & 14.4 \\
\hline & No & 76.3 & 70.3 & 85.6 \\
\hline \multirow[t]{2}{*}{ Enf. Respiratorias } & Sí & 54.9 & 59.1 & 50.8 \\
\hline & No & 45.1 & 40.9 & 49.2 \\
\hline \multirow[t]{2}{*}{ Carné de salud } & Sí & 54.1 & 76.9 & 80.2 \\
\hline & No & 45.9 & 23.1 & 19.8 \\
\hline \multirow[t]{2}{*}{ Matriculado guardería } & Sí & 0.6 & 15.5 & 37.5 \\
\hline & No & 99.4 & 84.5 & 62.6 \\
\hline
\end{tabular}




\begin{tabular}{lcccc} 
Lactancia correcta & Sí & 20.9 & 22.2 & 15.9 \\
Cuidado madre & No & 79.1 & 77.8 & 84.1 \\
& Sí & 77.5 & 82.2 & 68.1 \\
Trabaja madre & No & 22.5 & 17.8 & 31.9 \\
& Sí & 89.6 & 82.7 & 75.2 \\
Deporte madre & No & 10.4 & 17.3 & 24.8 \\
& Sí & 1.9 & 4.3 & 16.5 \\
Orden de nacimiento & No & 98.1 & 95.8 & 83.5 \\
Edad meses del menor & Media & 5.1 & 4.0 & 3.0 \\
Educación madre & Media & 30.4 & 31.1 & 30.3 \\
Edad madre & Media & 5.1 & 7.5 & 10.2 \\
\hline
\end{tabular}

Fuente: Encuesta de Condiciones de Vida 1999, 2006, 2014.

\section{RESULTADOS Y DISCUSIÓN}

El Ecuador se encuentra en una crisis de

Fig. 1. La malnutrición en Bolívar malnutrición infantil al ser comparado con los países de la región latinoamericana (FAO 2015). Las tasas de desnutrición crónica infantil sobrepasan el doble del promedio de la región que se ubica en $9 \%$, y las tasas de sobrepeso infantil superan el promedio latinoamericano que se ubica en 7\% (FAO 2020). La tendencia de reducción ha sido limitada en desnutrición, y el sobrepeso se ha incrementado notablemente (Freire et al. 2018; Rivera 2019). Dentro del país, existen casos alarmantes, como la provincia de Bolívar (MCDS 2010).

El panorama de la malnutrición infantil en la provincia de Bolívar es crítico en relación a las demás provincias del país (Rivera, Olarte y Rivera 2020). Como se indica en la Fig. 1, la desnutrición tiene una reducción importante, llegando a $35 \%$ en el año 2018, mientras que el sobrepeso, presenta un incremento sostenido, alcanzando una prevalencia de 19 $\%$.



Fuente: Elaboración propia

En la Tabla 2 se presentan los principales resultados del modelo de desnutrición infantil en Bolívar. Entre los factores que afectan a la desnutrición infantil se encuentran la pobreza, el bajo peso, y la educación de la madre. La pobreza tiene una asociación positiva con la desnutrición, donde la probabilidad de tener desnutrición se incrementa en $17 \%$ al pertenecer a un hogar pobre; este hecho 
tiene concordancia con la desigualdad dentro de la provincia y las dificultades dentro de los hogares pobres (CONCOPE 2006). El bajo peso tiene una relación positiva con la desnutrición, donde la probabilidad de tener desnutrición se incrementa en $39 \%$ al haber nacido con bajo peso. La educación de la madre tiene relación negativa con la desnutrición, donde la probabilidad de tener desnutrición se reduce en $4 \%$ al incrementarse la educación de la madre.

Tabla 2.- Determinantes de la desnutrición infantil en Bolívar

\begin{tabular}{|c|c|c|c|c|c|c|}
\hline \multirow{2}{*}{$\begin{array}{c}\text { Desnutrición } \\
\text { Variables }\end{array}$} & \multicolumn{6}{|c|}{ Años } \\
\hline & 1999 & & 2006 & & 2014 & \\
\hline \multirow[t]{2}{*}{ Rural } & 0.86 & $* * *$ & 0.17 & & -0.06 & \\
\hline & $(0.05)$ & & $(0.17)$ & & $(0.14)$ & \\
\hline \multirow[t]{2}{*}{ Pobre } & 0.76 & $* * *$ & 0.22 & $*$ & 0.17 & $* *$ \\
\hline & $(0.12)$ & & $(0.11)$ & & $(0.08)$ & \\
\hline \multirow[t]{2}{*}{ Agua red pública } & & & 0.18 & & -0.08 & \\
\hline & & & $(0.21)$ & & $(0.08)$ & \\
\hline \multirow[t]{2}{*}{ Servicio sanitario alc. } & 0.66 & $* * *$ & -0.01 & & 0.01 & \\
\hline & $(0.07)$ & & $(0.21)$ & & $(0.14)$ & \\
\hline \multirow[t]{2}{*}{ Recolección basura mun. } & & & -0.19 & & -0.06 & \\
\hline & & & $(0.17)$ & & $(0.09)$ & \\
\hline \multirow[t]{2}{*}{ Techo adecuado } & -0.30 & & 0.01 & & 0.07 & \\
\hline & $(0.26)$ & & $(0.11)$ & & $(0.08)$ & \\
\hline \multirow[t]{2}{*}{ Paredes adecuadas } & 0.01 & & 0.00 & & -0.02 & \\
\hline & $(0.20)$ & & $(0.08)$ & & $(0.08)$ & \\
\hline \multirow[t]{2}{*}{ Piso adecuado } & 0.36 & & -0.08 & & 0.03 & \\
\hline & $(0.09)$ & & $(0.17)$ & & $(0.14)$ & \\
\hline \multirow[t]{2}{*}{ Vitamina A } & & & 0.09 & & -0.24 & \\
\hline & & & $(0.08)$ & & $(0.16)$ & \\
\hline \multirow[t]{2}{*}{ Hierro } & -0.57 & $*$ & 0.08 & & 0.24 & \\
\hline & $(0.21)$ & & $(0.10)$ & & $(0.14)$ & \\
\hline \multirow[t]{2}{*}{ Edad } & 0.01 & $* * *$ & 0.00 & & 0.00 & \\
\hline & $(0.00)$ & & $(0.00)$ & & $(0.00)$ & \\
\hline \multirow[t]{2}{*}{ Mujer } & -0.03 & & -0.21 & $* * *$ & 0.02 & \\
\hline & $(0.11)$ & & $(0.07)$ & & $(0.06)$ & \\
\hline \multirow[t]{2}{*}{ Bajo peso } & & & -0.28 & & 0.39 & $* * *$ \\
\hline & & & $(0.26)$ & & $(0.10)$ & \\
\hline \multirow[t]{2}{*}{ Orden nacimiento } & 0.00 & & 0.05 & $* *$ & -0.03 & \\
\hline & $(0.05)$ & & $(0.02)$ & & $(0.02)$ & \\
\hline \multirow[t]{2}{*}{ Diarrea } & 0.02 & & -0.04 & & 0.03 & \\
\hline & $(0.15)$ & & $(0.07)$ & & $(0.09)$ & \\
\hline \multirow[t]{2}{*}{ Enf. Respiratorias } & 0.33 & $* *$ & -0.02 & & -0.09 & \\
\hline & $(0.13)$ & & $(0.08)$ & & $(0.06)$ & \\
\hline Carné salud & 0.22 & $*$ & -0.23 & $* * *$ & -0.01 & \\
\hline
\end{tabular}


$(0.13)$

Matriculado guardería

\begin{tabular}{|c|c|}
\hline Edad madre & $\begin{array}{c}-0.01 \\
(0.01)\end{array}$ \\
\hline Educación madre & $\begin{array}{l}-0.07 \\
(0.02)\end{array}$ \\
\hline Lactancia correcta & $\begin{array}{l}-0.23 \\
(0.18)\end{array}$ \\
\hline Cuidado madre & $\begin{array}{c}0.14 \\
(0.17)\end{array}$ \\
\hline Trabaja madre & $\begin{array}{c}0.13 \\
(0.28)\end{array}$ \\
\hline \multicolumn{2}{|l|}{ Deporte madre } \\
\hline $\mathrm{N}$ & 115 \\
\hline Pseudo R2 & 0.40 \\
\hline Prob. Neta Predicha & 0.85 \\
\hline \multicolumn{2}{|c|}{$\begin{array}{l}\text { Encuesta de Condiciones de Vida 1999, 2006, } 2 \\
\text { antil. Nota: * significativo al } 90 \% \text { de confianza; }\end{array}$} \\
\hline \multicolumn{2}{|c|}{$\begin{array}{l}\text { abla } 3 \text { se presentan los principales } \\
\text { os del modelo de sobrepeso infantil } \\
\text { var. Entre los factores que afectan } \\
\text { epeso infantil se encuentran la } \\
\text { ilidad de agua por red pública, el } \\
\text { salud y el trabajo de la madre. El }\end{array}$} \\
\hline
\end{tabular}

En la Tabla 3 se presentan los principales resultados del modelo de sobrepeso infantil en Bolívar. Entre los factores que afectan al sobrepeso infantil se encuentran la disponibilidad de agua por red pública, el carné de salud y el trabajo de la madre. El agua por red pública tiene una relación negativa con la presencia de sobrepeso,

\begin{tabular}{cc}
$(0.08)$ & $(0.09)$ \\
-0.05 & 0.09 \\
$(0.10)$ & $(0.09)$ \\
-0.01 & 0.01 \\
$(0.01)$ & $(0.01) \quad$ \\
0.00 & $-0.04 \quad * * *$ \\
$(0.01)$ & $(0.01)$ \\
-0.02 & -0.09 \\
$(0.10)$ & $(0.09)$ \\
-0.13 & 0.06 \\
$(0.09)$ & $(0.09)$ \\
0.04 & -0.05 \\
$(0.11)$ & $(0.09)$ \\
-0.23 & -0.11 \\
$(0.16)$ & $(0.09)$ \\
267 & 320 \\
0.17 & 0.20 \\
0.68 & 0.71 \\
\hline
\end{tabular}

Fuente: Encuesta de Condiciones de Vida 1999, 2006, 2014. La variable dependiente es binaria acerca de desnutrición crónica infantil. Nota: * significativo al 90\% de confianza; ** significativo al 95\% de confianza; *** significativo al $99 \%$ de confianza.

reduciendo la probabilidad de padecerla en $12 \%$, lo cual está asociado a la disponibilidad de agua segura y la reducción de problemas de parásitos. El disponer de un carné de salud y el que la madre trabaje tienen una relación negativa con el sobrepeso, reduciendo la prevalencia en $8 \%$ y $9 \%$, respectivamente, lo cual podría estar asociado con el seguimiento del menor de cinco años.

Tabla 3.- Determinantes del sobrepeso infantil en Bolívar

\begin{tabular}{|c|c|c|c|c|c|}
\hline Sobrepeso & & & Años & & \\
\hline Variables & 1999 & & 2006 & 2014 & \\
\hline \multirow[t]{2}{*}{ Rural } & & & & -0.10 & \\
\hline & & & & $(0.12)$ & \\
\hline \multirow[t]{2}{*}{ Pobre } & 0.00 & & 0.00 & -0.05 & \\
\hline & $(0.00)$ & & $(0.07)$ & $(0.05)$ & \\
\hline \multirow[t]{2}{*}{ Agua red pública } & & & & -0.12 & $* *$ \\
\hline & & & & $(0.05)$ & \\
\hline \multirow[t]{2}{*}{ Servicio sanitario alc. } & 1.00 & $* * *$ & & 0.03 & \\
\hline & $(0.00)$ & & & $(0.09)$ & \\
\hline Recolección basura mun. & & & & -0.05 & \\
\hline
\end{tabular}




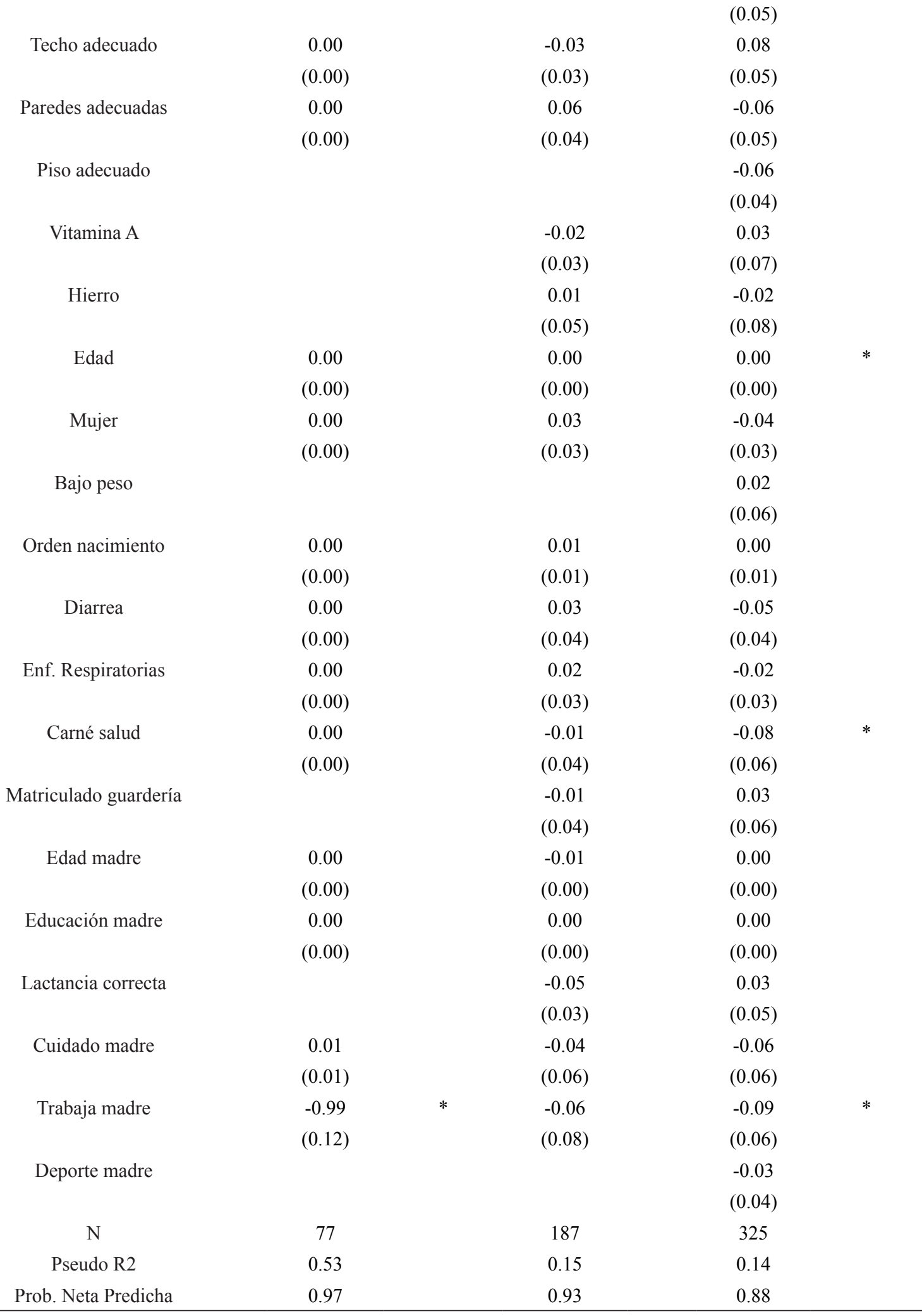

Fuente: Encuesta de Condiciones de Vida 1999, 2006, 2014. La variable dependiente es binaria acerca de sobrepeso infantil. Nota: * significativo al $90 \%$ de confianza; $* *$ significativo al $95 \%$ de confianza; $* * *$ significativo al $99 \%$ de confianza.

Con estos resultados, la provincia de Bolívar dependen de su contexto. Los hallazgos de denota unos determinantes particulares de la provincia son distintos a lo encontrado en la desnutrición y el sobrepeso infantil que distintas provincias del Ecuador como Carchi, 
Chimborazo, Esmeraldas, Imbabura, Manabí y Santa Elena (Rivera, Olarte y Rivera 2020; Rivera y Olarte 2020 y Rivera 2019). En los distintos estudios se encuentra que los determinantes son propios de cada provincia, no son estándares para el país.

Ante ello, es fundamental que se reflexione sobre la pertinencia para el Ecuador y la provincia de Bolívar de una política pública contingente, que tenga flexibilidad hacia el contexto, que ataque la desnutrición infantil y también prevenga el sobrepeso. En este proceso, los resultados de esta investigación son relevantes para una toma de decisiones informada. En suma, la crisis de nutrición infantil en el Ecuador requiere de una política pública que actúe como un "instrumento de transformación de la sociedad" (Rivera 2019).

\section{CONCLUSIONES}

Bolívar presenta un caso crítico de malnutrición infantil. Entre los principales determinantes se encuentran factores ambientales, sociales y personales que son particulares para esta provincia. Por un lado, la desnutrición se asocia con la pobreza, el bajo peso y la educación de la madre; en esa línea, la desnutrición está afectada por limitaciones básicas, donde Bolívar presenta niveles de pobreza sobre el promedio nacional; por su parte, el sobrepeso se relaciona con la disponibilidad de agua por red pública, carné de salud y trabajo de la madre; en ese sentido, el sobrepeso está afectado por temas de acceso a servicios. Esta investigación visibiliza un problema crítico de Bolívar. Las potencialidades del estudio están en que se podrían desarrollar políticas públicas enfocadas en el mejoramiento de la malnutrición infantil. Para ello, es vital el trabajo en la reducción de la pobreza, desde una perspectiva multidimensional, el mejoramiento de capacidades y oportunidades educativas, así como una mayor provisión de servicios de salud para realizar un seguimiento oportuno de la madre gestante y del recién nacido.

\section{BIBLIOGRAFÍA}

Caulfield, L., De Onis, M., Blossner, M., \& Black, R. (2004). "Undernutrition as an Underlying Cause of Child Deaths Associated with Diarrhea, Pneumonia, Malaria, and Measles." The American Journal of Clinical Nutrition, 193-98.

Consorcio de Consejos Provinciales del Ecuador (CONCOPE). (2006). Objetivos de Desarrollo Del Milenio, Estado de Situación 2006: Provincia de Bolívar. Quito: CONCOPE.

Food and Agriculture Organization (FAO). (2020). Panorama de La Inseguridad Alimentaria En América Latina y El Caribe 2020. Roma: FAO.

FAO. (2015). Panorama de La Inseguridad Alimentaria En América Latina y El Caribe 2015. Roma: FAO.

Freire, W., Waters, W., Rivas-Mariño, G., \& Belmont, P. (2018). “The Double Burden of Chronic Malnutrition and Overweight and Obesity in Ecuadorian Mothers and Children, 1986-2012." Nutrition and Health 1-8. 
Gillespie, S., McLachlan, M., \& Shrimpton, R. (2003). Combating Malnutrition: Time to Act. Washington: World BankUNICEF.

Gobierno de la provincia de Bolívar. (2015).

Plan de Desarrollo y Ordenamiento Territorial. Guaranda: Gobierno de la provincia de Bolívar.

Gutiérrez, N., Ciuffardi, T., Rokx, C., Brousset, H., \& Gachet, N. (2017). Apuntando Alto: Retos de La Lucha Contra La Desnutrición Crónica En Ecuador. Washington: Banco Mundial.

Instituto Nacional de Estadísticas y Censos (INEC). (2014). Mapa de Pobreza y Desigualdad Por Consumo. Quito: INEC.

INEC. (2015). Informe de Resultados ECV 2013-2014. Quito: INEC.

Martorell, R. (1999). The Nature of Child Malnutrition and Its Long-Term Implications. Food and Nutrition Bulletin 20(3): 5.

Ministerio Coordinador de Desarrollo Social (MCDS). (2010). Mapa de Desnutrición Crónica En El Ecuador. Quito: MCDS.

MCDS. (2017). Informe de Desarrollo Social 2007-2017. Quito: MCDS.

Organización de las Naciones Unidas (ONU). (2015). Hambre Cero: Por Qué Es Importante. New York: ONU.

ONU. (2019). Informe de Los Objetivos de Desarrollo Sostenible. New York: ONU.

Rivera, J., Olarte S., \& Rivera, N. (2020). La malnutrición infantil en Esmeraldas: ¿un éxito relativo? La U Investiga 7(2): 68-
78.

Rivera, J., Olarte S., \& Rivera, N. (2020). Madre hay una sola: la malnutrición infantil en Carchi. Sathiri-Sembrador 15(2): 291-300.

Rivera, J., Olarte S., \& Rivera, N. (2020). Las secuelas del terremoto: el problema de la malnutrición infantil en Manabí. Revista ECA Sinergia 11(3): 40-49.

Rivera, J. \& Olarte S. (2020). La evolución de la malnutrición infantil en Chimborazo: Entre progresos y desafíos. Ciencia al Servicio de la Salud y Nutrición 11(1): 33-43.

Rivera, J., Olarte S., \& Rivera, N. (2020). Crónica de una muerte anunciada: la malnutrición infantil en Santa Elena. Ciencias Pedagógicas e Innovación 8(1): 28-35.

Rivera, J., Olarte S., \& Rivera, N. (2020). Evolución y factores determinantes de la malnutrición infantil en Imbabura. La U Investiga 6(2): 8-18.

Rivera, J. (2019). Las políticas públicas y la gestión pública: un análisis desde la teoría y la práctica, entrevista a AndréNoël Roth. Estudios de la Gestión 5(1): 223-29.

Rivera, J. (2019). La malnutrición infantil en Ecuador: una mirada desde las políticas públicas. Estudios de Políticas Públicas 5(1):89-107.

Rivera, J. (2019). La malnutrición en Santa Elena: una mirada multidimensional. Ciencias Pedagógicas e Innovación 7(1): 104-111. 
Rivera, J. (2019). La malnutrición

en Chimborazo: una mirada

multidimensional. Ciencia al Servicio de la Salud y Nutrición 10(1): 2-12.

Rivera, J. (2012). Obesidad En México: Recomendaciones Para Una Política de Estado. México: UNAM.

Rivera, J., Pedraza, L., Martorell, R., \& Gil, A. (2014). Introduction to the Double Burden of Undernutrition and Excess Weight in Latin America. The American Journal of Clinical Nutrition 100:1613S$6 \mathrm{~S}$.

United Nations Children's Emergency Fund (UNICEF). (2013). Improving Child Nutrition: The Achievable Imperative for Global Progress. New York: UNICEF.

World Health Organization (WHO). (2000). Obesity: Preventing and Managing the Global Epidemic: Report of a WHO Consultation. Geneva: World Health Organization.

WHO. (2006). Child Growth Standards. Geneva: WHO. 